\title{
Introducción al dossier \\ Etnografía y educación: \\ estudios colaborativos \\ con niños, niñas y jóvenes
}

Ethnography and Education: Collaborative research

with children and young people

doi: 10.11144/Javeriana.m13.idee

Alba Lucy Guerrero

alba.guerrero@javeriana.edu.co

Pontificia Universidad JaVeriana, Colombia

ORCID: https://orcid.org/0000-0003-1605-2983

Diana Milstein

diana.milstein4@gmail.com

CIS-Conicet/Instituto de Desarrollo Económico y Social, Argentina

(iD) ORCID: https://orcid.org/0000-0002-5261-2194

Para citar este artículo | To cite this article

Guerrero, A. L. \& Milstein, D. (2020). Introducción al dossier Etnografía y educación: estudios colaborativos con niños niñas y jóvenes. magis, Revista Internacional de Investigación en Educación, 13, 1-12. doi: 10.11144/Javeriana. m13.idee 
Este dossier presenta trabajos de campo y resultados de investigaciones etnográficas en colaboración con niñas, niños y jóvenes (NNJ) realizados en contextos educativos escolares y no escolares para analizar las incidencias e impactos de la etnografía en colaboración con NNJ en el campo de la investigación educativa. Los artículos aquí presentados abren oportunidades de diálogo y debate sobre algunos de los temas y problemas actuales planteados por los discursos académicos y políticos sobre educación, tales como políticas, identidades, prácticas culturales y lingüísticas, geografías y migraciones en contextos locales. Estos asuntos se abordan de manera situada teniendo en cuenta las particularidades sociohistóricas de cada lugar. Los once artículos que componen este dossier son contribuciones de investigadores que han participado en eventos académicos organizados por la Red Internacional de Etnografía con Niños, Niñas, Adolescentes y Jóvenes (RIENN). El trabajo de la RIENN incluye más de diez años de investigación e intercambio entre etnógrafxs y NNJ en contextos diversos situados en regiones de Latinoamérica, Norteamérica y Europa, algunos de ellos organizados por la Facultad de Educación de la Pontificia Universidad Javeriana.

Lxs autores de los artículos que presentamos se desempeñan en instituciones de Argentina, Australia, Brasil, Colombia, España, Estados Unidos, México, Nueva Zelanda e Inglaterra, y se nutren de diferentes ámbitos disciplinares, que incluyen educación, lingüística, antropología, psicología, sociología, economía, geografía, estudios sociales de la infancia, estudios culturales y comunicación. Los textos son resultado de estudios realizados en colaboración con NNJ en entornos educativos urbanos y rurales, reunidos con la intención de poner en diálogo diferentes discursos y prácticas atravesados por categorías analíticas y sociales en función de expandir las posibilidades teóricas y metodológicas de la investigación colaborativa con NNJ en entornos educativos.

La tendencia a desarrollar etnografías con NNJ y el reconocimiento de que son interlocutores válidos proviene de un debate que inicia en los años ochenta en el interior de los campos académicos en torno al estatus que se les otorga a Ixs niñxs como sujetos sociales con capacidad de agencia para involucrarse en las dinámicas sociales, políticas y culturales de los diversos escenarios en los que actúan.

1 Haremos uso de la letra $x$ para referirnos a grupos de personas cuya adscripcion de género o identificación con un pronombre en particular no se conozca. Se considera que la utilización de la $x$ es una posibilidad de producción de lenguaje no binario. De este modo, con su uso nos distanciamos de aquel lenguaje que remite al mal llamado "genérico" masculino, como del uso del -os/-as, que también colabora con la definición de binarismos. 
En los trabajos que conforman este dossier Ixs lectores encontrarán estilos, formas y particularidades de colaboración entre etnógrafos y NNJ, diversas modalidades de relaciones que surgen y se despliegan durante los procesos de investigación, maneras novedosas de resolver desafíos epistemológicos, metodológicos y de representación de resultados y múltiples aperturas que invitan a expandir debates en la investigación educativa en general, en la etnografía colaborativa y educativa, y en los estudios sociales sobre infancias y juventudes.

Tomando como punto de partida que la participación de sujetos y pobladores que habitan los lugares donde se realiza el trabajo de campo es una parte integral de todos los estudios etnográficos, los textos de esta compilación se ocupan de la manera en que la colaboración entre NNJ y expertxs académicxs en las investigaciones impactan el campo de la etnografía educativa, así como la comprensión de fenómenos y procesos relacionados con la educación y los contextos socioculturales. Lxs autorxs de los artículos coinciden en considerar a la etnografía colaborativa como una forma de producción de conocimiento que no se restringe a sus aspectos técnicos/metodológicos, sino que los incluye en una trama de "relaciones coetáneas que estimulan el flujo intersubjetivo y fomentan la coproducción entre investigadoras e investigadores académicos y no académicos" (Milstein \& Guerrero, 2021). De este modo, el abordaje colaborativo reconoce explícitamente que los sujetos no académicos y los investigadores son coproductores y coautores de un proceso de generación de conocimiento etnográfico. La colaboración abarca el conjunto total de tareas involucradas en el proceso de investigación o algunas de ellas, y da lugar a incorporar, tanto para Ixs interlocutores como para Ixs investigadores, nuevas herramientas conceptuales que otorgan nuevos sentidos a las realidades contemporáneas (Milstein, Clemente \& Guerrero, 2019; Rappaport, 2004). De manera que las categorías sociales en este tipo de investigación son elaboradas con los NNJ y no preescritas por los marcos teóricos de los investigadores (Alegre \& Gandulfo, 2020; Guerrero, Clemente, Dantas-Whitney \& Milstein, 2017).

Las modalidades de colaboración presentadas en los textos son diversas y aparecen en diferentes momentos de la investigación — problematización, trabajo de campo, análisis y escritura. Maicol Ruiz-Morales (2021) muestra en su artículo cómo la interlocución en la problematización con un grupo de estudiantes y maestros que ya venían trabajando en colaboración en la escuela les implica al grupo y al investigador repensar sus preguntas de investigación. Ruiz-Morales afirma que "aquel ejercicio implicó, parafraseando a Guber (2005, p. 39), que tanto los miembros del semillero como yo reconociéramos la alteridad que encarnábamos cuando nos poníamos 
en situación como investigadores". En términos de la colaboración en el trabajo de campo, observamos experiencias como las de Maria Dantas-Whitney (2020) y Silvina Fernández (2021), en las que el flujo intersubjetivo de comunicación entre niñxs de primaria y las investigadoras tiene un impacto en el planteamiento inicial de las investigaciones y también en la manera de comprender el ámbito educativo y los procesos escolares. Dantas-Whitney inicia su estudio con el propósito de identificar las prácticas e interacciones situadas en el aula que fomentan oportunidades de aprendizaje productivas para niñxs bilingües. Sin embargo, la estrategia de escribir un diario de campo con Ixs niñxs y sus familias, propuesta por la investigadora y la maestra, se convierte en un

vehículo importante para que los niñxs expresen sus formas cotidianas de conocimiento, para validar el capital lingüístico y cultural de sus hogares y comunidades, y para transformar el plan de estudios escolar oficial por un plan de estudios negociado y coconstruido (p. 9).

De manera similar, Fernández (2021, p. 21) muestra en su estudio cómo "la visión de Ixs niñxs aporta elementos sustanciales para replantear el tema de la alimentación en un contexto escolar, en general, y del comedor escolar, en particular". El análisis de las interacciones con Ixs niñxs sobre la alimentación en la escuela evidencia que las prácticas de comensalidad se rigen por lógicas escolares que priorizan disputas o aspectos organizativos que afectan la calidad de su alimentación.

Alegre \& Gandulfo (2020) comparten en su artículo los resultados producidos durante el proceso de análisis de los registros de observación realizados por estudiantes de secundaria sobre los usos lingüísticos y sus significaciones en un contexto bilingüe guaraní-castellano. A partir del análisis colaborativo de dos situaciones etnográficas, Ixs estudiantes proponen la categoría "hablar en bilingüe" para caracterizar algunos de los repertorios lingüísticos observados. La creación de la categoría nativa hablar en bilingüe aporta una perspectiva original para describir los repertorios lingüísticos estudiados. Por su parte, Barley (2020) diseña una herramienta de análisis participativo, Participatory Analysis Tool (PAT), para que Ixs niñxs que toman parte junto con la investigadora compartan el análisis a través de la revisión conjunta de narrativas producidas por ellos.

En una línea similar, Di Caudo-Villoslada (2020) muestra cómo, en el proceso de escritura y publicación de un libro con estudiantes universitarios, los intercambios y la toma de decisiones sobre aspectos como el título del libro y el propio proceso de publicación dieron lugar a la producción de nuevos significados entre interlocutores e investigadora y a replantear las 
perspectivas relativas a las posiciones de estudiantes indígenas y afrodescendientes en el ámbito de la educación superior universitaria.

Un tema que resalta en este conjunto de artículos como aporte singular a la investigación educativa es la horizontalidad en las relaciones entre adultos y niños que se construye en la experiencia investigativa colaborativa en contextos educativos. En ese sentido entendemos que los artículos tienen un impacto no solo para la investigación, sino también para los contextos educativos. Por ejemplo, Ruth Barley (2020) muestra que el poder que ganaron Ixs niñxs de un preescolar al incidir en su investigación y tomar decisiones para la recolección y el análisis de la información impactó reduciendo asimetrías existentes entre niñas, niños y adultos en la cotidianidad de la vida escolar. El proyecto permitió que Ixs niñxs enseñaran a los adultos, revirtiendo, así, aspectos de la relación escolar entre enseñante-enseñado y retando algunos de los discursos dominantes sobre el lugar social de subordinación de Ixs niñxs. Con una perspectiva similar, Huerta-Cordova, Clemente-Olmos \& Córdova-Hernández (2021), en su investigación con jóvenes, analizan la producción de un texto académico en coautoría entre estudiantes y docentes. Sostienen que el ejercicio de escritura colaborativa hizo posible un cambio de situación en las relaciones de poder establecidas en los ambientes universitarios al modificar la relación vertical de autoridad por parte del profesorado hacia una interacción más horizontal, lo que permitió generar conocimiento genuino y original sobre el contexto en que transcurre la vida escolar. Este modo de analizar el contexto es profundizado también por Machado e Silva (2021), quien a través de la presentación de un saber común de niñas y niños muestra hasta qué punto el contexto local no solo está en la escuela, sino que es allí donde se vuelve impensadamente visible.

\section{Presentación de los textos}

De los once textos que conforman este dossier, ocho fueron elaborados a partir de estudios empíricos que muestran formas creativas de producir datos de manera colaborativa con los NNJ de diversos grupos raciales, étnicos y socioeconómicos en países diferentes, haciendo visible la incorporación de sus perspectivas (Alegre \& Gandulfo, 2020; Barley, 2020; Dantas-Whithney, 2020; Di Caudo-Villoslada, 2020; Fernández, 2021; Huerta-Cordova et al., 2021; Ruiz-Morales, 2021; Machado e Silva, 2021). Otros dos artículos incluyen un estado del arte y una reflexión. El primero, a través de la revisión de literatura sobre prácticas escolares y de juego de un pueblo indígena en Australia, muestra las diferencias en concepciones de niñez dependiendo de los contextos culturales, sociales y económicos (Holmes, 2020). El otro 
analiza de manera minuciosa cuatro trabajos etnográficos latinoamericanos que dan cuenta de procesos de investigación con NNJ, exponiendo cómo una lectura crítica del trabajo colaborativo realizado por otros investigadores puede nutrir la investigación educativa (Milstein \& Guerrero, 2021). Finalmente, este dossier presenta la reseña de un libro que reúne una selección de estudios etnográficos en contextos educativos desarrollados en colaboración con NNJ (Bácares-Jara \& Bertoli, 2021). Este dossier se presentará repartido en dos volúmenes consecutivos.

\section{Primera parte del dossier (2020)}

Ruth Barley, en su trabajo "'Why have you not written my name?': Collaborative research with children", muestra como niñxs de una escuela preescolar de Inglaterra negocian su participación en la investigación a través de la escritura en los procesos de recolección de datos y de análisis. Lxs niñxs, que en su mayoría provienen de grupos culturales minoritarios en Inglaterra, producen sus propios datos visuales y escritos, y participan con la investigadora en el análisis y reflexión sobre los resultados de la investigación. Este trabajo contribuye a la investigación educativa, al evidenciar cómo la participación activa de lxs niñxs en los procesos de investigación facilita un mayor nivel de consentimiento informado, una puesta en cuestión de facetas de las relaciones entre niñxs y adultos, y una visión más profunda del mundo social de Ixs niñxs. Los resultados de este artículo tienen relevancia para el desarrollo de procesos de enseñanza y aprendizaje en la medida en que la producción de los textos escritos y visuales permitió a niñas y niños, que en su mayoría están aprendiendo inglés, oportunidades para reflexionar sobre su proceso de alfabetización y consolidar sus aprendizajes escolares.

Tamara Alegre y Carolina Gandulfo, en su artículo "'Hablan en bilingüe': repertorios lingüísticos guaraní-castellano en Corrientes, Argentina", presentan una investigación en colaboración sobre los repertorios lingüísticos bilingües guaraní-castellano de una escuela secundaria rural de Corrientes, Argentina. El estudio elabora una caracterización sociolingüística a partir de los registros de observación de eventos comunicativos realizados por los jóvenes en sus casas. A través de dos situaciones etnográficas ocurridas en la escuela en el marco de actividades de análisis, el estudio muestra la manera en que la metodología colaborativa permite un diálogo entre categorías teóricas y nativas, tal como lo expresa el enunciado "hablar en bilingüe", que aporta una perspectiva original para describir los repertorios lingüísticos estudiados. En términos de la investigación educativa, el diseño metodológico del proyecto de investigación se articula con los propósitos pedagógicos de un espacio curricular llamado plan de búsqueda. En este 
sentido, el aporte a la investigación educativa supone la problematización de prácticas lingüísticas y sus sentidos en el marco de un proceso de formación pedagógico que incluye en sus actividades educativas herramientas de la investigación social.

El artículo titulado "'Comimos quesadillas y después jugamos tag:' Children's bilingual/bicultural identity in a U. S. elementary school", de Maria Dantas-Whitney es el resultado de un estudio etnográfico colaborativo realizado con niñxs oriundos de países de Latinoamérica en una clase bilingüe de escuela primaria en una comunidad rural en los Estados Unidos. Se utilizó para ello una actividad realizada en clase: un diario de familia escrito por Ixs niñxs en colaboración con sus parientes. A través del análisis de las narrativas puestas en juego en esos diarios, el estudio buscó comprender cómo la fusión creativa de los discursos del hogar y de la escuela permitieron que niñxs e investigadoras examinen, validen y expandan sus identidades bilingües y biculturales. Este trabajo aporta a la investigación educativa una exploración colectiva de historias, instituciones, prácticas, relaciones, geografías y futuros imaginados construidos con Ixs niñxs, quienes también desarrollan su sentido de identidad y agencia a través de conexiones con las historias pasadas y presentes de sus comunidades. En cuanto a la etnografía colaborativa, esta contribuye a pensar cómo prácticas y conocimientos desplegados junto con Ixs niñxs en las clases pueden fomentar procesos de coinvestigación.

María Verónica di Caudo-Villoslada, en su artículo "'No estamos de acuerdo con el título'. Escribir un libro con estudiantes universitarios interculturales", presenta un proceso de investigación, escritura y publicación de un libro entre un grupo de investigadores conformado por estudiantes universitarixs y una académica. La experiencia ocurre en Ecuador, en el contexto de una investigación etnográfica sobre procesos de inclusión de jóvenes a los estudios superiores. El estudio muestra cómo el trabajo colaborativo, a través de talleres con Ixs estudiantes, permitió descubrir los sentidos, significados y prácticas que los convertían en "estudiantes interculturales", que desafiaban su posición como "beneficiarios" de políticas y programas inclusivos. Las interacciones de lxs jóvenes evidencian identidades dinámicas y flexibles que visibilizan la "diferencia" como un lugar potente de "reconocimiento" en distintas situaciones, entornos y acontecimientos. Los procesos de coautoría y coproducción de conocimiento en las áreas típicas de las universidades, academia, vinculación con la sociedad e investigación, evidencian la capacidad de Ixs estudiantes para resignificar, sostener la cultura universitaria y contribuir en el desarrollo de políticas y transformaciones prácticas en el campo de la educación superior. Asimismo, este estudio potencia formas de descentramiento necesarias en Ixs investigadores y abre 
oportunidades para cuestionar las categorías que tienden a predeterminar lo que se investiga y cómo se investiga en educación.

Catherine Holmes, en su artículo "Childhood, play and school: A literature review in Australia", presenta una revisión de literatura sobre la infancia, el juego y la escuela en contextos remotos habitados por las primeras naciones en Australia (pueblos indígenas). Toma como punto de partida la primera infancia en cuanto etapa fundamental del desarrollo infantil, se centra en la clasificación de esta para los pueblos aborígenes e isleños del estrecho de Torres, en Australia, y describe las formas en que Ixs niñxs adquieren prácticas sociales y culturales. De acuerdo con el estudio, aún se tiene un conocimiento limitado sobre el desarrollo infantil de niñxs de cero a siete años en las primeras naciones. El estudio pone en evidencia la brecha entre las normas educativas y de salud en las poblaciones infantiles de Ixs niñxs ngaanyatjarra y pintupi, y sus familias, mostrando que las experiencias de aprendizaje de lxs niñxs, tanto dentro como fuera de la escuela, proporcionan efectos profundos en su rendimiento y sus desempeños sociales. Así, esta revisión brinda oportunidades para mostrar formas innovadoras en que los contextos de aprendizaje fuera de la escuela consolidan el aprendizaje en el entorno escolar.

\section{Segunda parte del dossier (2021)}

El texto de Diana Milstein y Alba Lucy Guerrero, titulado "Lecturas de etnografías colaborativas con niñas, niños y jóvenes en contextos educativos latinoamericanos", es un artículo de reflexión a partir del análisis de cuatro investigaciones etnográficas colaborativas con NNJ realizadas en países latinoamericanos. Las autoras reconstruyen la producción compartida de conocimiento entre etnógrafos y NNJ, y dan cuenta del aporte de estos trabajos al campo de la investigación educativa y de la comprensión de los contextos sociales y culturales en los que se desarrollaron esas etnografías. El trabajo pone en discusión perspectivas dominantes en la investigación educativa que tienden a excluir o subsumir el protagonismo de la mayoría de los actores, minimizando, así, el conocimiento local imprescindible para desarrollar nuevas y originales comprensiones de procesos educativos, y aporta estrategias para desarrollar trabajo de campo y de escritura entre etnógrafos y NNJ.

El trabajo investigativo "'Nos convidamos solos en un cuento que no era nuestro': encuentros con niños investigadores", realizado por Maicol Mauricio Ruiz-Morales, tiene como objetivo indagar desde adentro los procesos de valorización de la caficultura de montaña en un municipio colombiano. Metodológicamente, el estudio parte de reconocer y valorar la preexistencia de procesos investigativos de base en el seno de las 
comunidades escolares, que buscan realizar su valor potencial participando de procesos de apropiación patrimonial del Paisaje Cultural Cafetero (PCC). De este modo, el investigador no es quien incorpora a Ixs niñxs y jóvenes en su investigación, sino que participa de una suerte de plataforma interdisciplinaria e intergeneracional compartida con estudiantes y docentes locales, expertos de la comunidad, al igual que con investigadores y estudiantes universitarios. El autor reconstruye de manera etnográfica los procesos investigativos en colaboración, no con la intención de validarlos, sino como medio para descubrir reflexivamente los significados y sentidos que NNJ y adultos producen como nativos sobre sí mismos, sobre los demás y lo que ocurre en su entorno. La investigación presenta argumentos que amplían la comprensión teórica y situada, tanto de la valorización de la caficultura de montaña como del valor de las experiencias investigativas agenciadas desde las escuelas rurales. Esto deslindando las miradas que, desde la investigación educativa, han relacionado tradicionalmente la economía con la educación.

El artículo de Regina Coeli Machado e Silva, titulado "'A mala não é dela': saber das crianças em contexto escolar de fronteira", hace visible la existencia de un saber común entre Ixs niñxs de una escuela primaria de Foz de Iguazú, Brasil, en la frontera con Paraguay, sobre el modo de vida y de trabajo de los adultos. El título "A mala não é dela" ('La bolsa no es de ella') es la interpretación de uno de los niños sobre la escena final de un cortometraje que se presenta como parte de la investigación colaborativa con niñxs y adultos trabajadores y agentes de la escuela. El film muestra a una joven que estaba cruzando la frontera desde Paraguay hacia Brasil y es alertada por la presencia de la policía del lado de Brasil; ella inmediatamente se apresura hasta los muros bajos del puente que vincula ambos países y tira la bolsa que le habían entregado antes al río que corre debajo de ese puente. La autora argumenta que las reinvenciones que Ixs niñxs hicieron del relato audiovisual revelan que ellxs y comparten un saber local y público relativo a los riesgos del "trabajo ilegal, pero legítimo" de los adultos con quienes viven. El texto revela las posibilidades que ofrecen los contextos escolares como medio para estudiar temas fundamentales que involucran la vida de Ixs niñxs y que van más allá de la escolarización y del aprendizaje, enriqueciendo, así, la investigación educativa. Muestra también la importancia que reviste la colaboración de niñxs y adultos en los procesos investigativos.

Vilma Huerta-Cordova, Ángeles Clemente-Olmos y Lorena CórdovaHernández presentan una reflexión sobre la investigación etnográfica colaborativa dentro el salón de clases de una universidad estatal pública de la ciudad de Oaxaca, México. En su artículo "El contexto vivo: reflexiones sobre una experiencia etnográfica colaborativa universitaria", las autoras 
analizan los intercambios entre una profesora y sus estudiantes sobre la forma de escribir el contexto de sus estudios etnográficos y la construcción colaborativa de este proceso. El estudio evidencia la manera en que el trabajo en colaboración los lleva a descubrir que existe un contexto vivo, orgánico y fluido, que se conforma de una variedad de personas (que oficialmente no se consideran universitarios) y de sus puntos de vista sobre las diversas maneras de posicionarse en el ámbito universitario. Este trabajo aporta elementos prácticos para el proceso de formación de futuros profesores en investigación y más específicamente en etnografía educativa. En este tipo de experiencias investigativas Ixs estudiantes reconocen en la colaboración investigativa una manera más horizontal de generar conocimiento. En lo que respecta a los profesores universitarios, este artículo ilustra la forma en que la reflexión sobre la colaboración puede mostrar situaciones interpersonales que de otra forma no se trabajarían dentro de un salón de clases.

En el artículo "'iEs porque no le ponen onda!': prácticas de comensalidad en la escuela", Silvina Fernández expone un análisis sobre la perspectiva de Ixs niñxs en torno a la alimentación y las prácticas de comensalidad que se desarrollan en el comedor de una escuela pública estatal de la provincia de Buenos Aires, Argentina. Una conversación con dos niñas y la perplejidad que esta genera en la investigadora dan lugar a comprender situaciones que se suscitan en relación con el comedor y en torno a conflictos con los diversos actores que van más allá de los fenómenos alimentarios en sí. Este trabajo aporta una mirada crítica a los modos en los que se ha estudiado la alimentación en el contexto escolar, orientados a los fenómenos nutricionales. Asimismo, aporta argumentos contundentes a favor de la consideración de lxs niñxs como actores relevantes con quienes es posible desnaturalizar prácticas y lógicas escolares que empañan y obturan la comprensión de fenómenos escolares.

Finalmente, Camilo Bácares-Jara y Anny Bertoli presentan una reseña de la compilación Bordes, límites y fronteras. Encuentros etnográficos con niños, niñas y adolescentes, que complementa de manera pertinente este dossier. Los autores presentan los capítulos de este libro como una reflexión sobre las rupturas que provoca el acompañamiento de NNJ en la investigación educativa y como una oportunidad para repensar el concepto de otredad, planteando un reconocimiento de NNJ como otros capaces de interpelar las categorías preestablecidas por Ixs investigadores y de crear conocimiento y nuevas lecturas para comprender los fenómenos estudiados.

Como editoras de este dossier expresamos nuestro sincero agradecimiento a Ixs colegas que hicieron posible esta compilación de trabajos, al 
conjunto de colegas revisores y a Ixs integrantes de la dirección, el comité editorial y el equipo técnico de la revista magis.

\section{Sobre las autoras}

Alba Lucy Guerrero es profesora asociada de la Facultad de Educación de la Pontificia Universidad Javeriana, Colombia, y coordinadora de la línea de investigación Infancias, Cultura y Educación. Doctora en Educación de la Universidad de California, Santa Bárbara, Estados Unidos. Máster en Perspectivas Culturales en Educación de la Universidad de California, Santa Bárbara, Estados Unidos, y magíster en Desarrollo Educativo y social del Cinde-Universidad Pedagógica Nacional, Colombia. Miembro del comité académico y cofundadora de la Red Internacional de Investigación Etnográfica con Niños, Niñas, Adolescentes y Jóvenes (RIENN).

Diana Milstein es investigadora del Centro de Investigaciones Sociales (CIS) (Consejo Nacional de Investigaciones Científicas y Técnicas-Instituto de Desarrollo Económico y Social), profesora del Doctorado en Ciencias Sociales de la Universidad Nacional General Sarmiento y de la Maestría en Investigación Educativa del CEA, Universidad Nacional de Córdoba, Argentina. Es doctora en Antropología Social de la Universidad de Brasilia y magíster en Antropología Social de la Universidad Nacional de Misiones. Es cofundadora y coordinadora general de la Red Internacional de Etnografía con Niños, Niñas, Adolescentes y Jóvenes (RIENN).

\section{Referencias}

Alegre, T. D. \& Gandulfo, C. (2020). "Hablan en bilingüe": repertorios lingüísticos guaraní-castellano en Corrientes, Argentina. magis, Revista Internacional de Investigación en Educación, 13, 1-25. doi: 10.11144/Javeriana.m13.hbrl

Bácares-Jara, C. \& Bertoli, A. (2021). Reseña del libro Bordes, límites y fronteras. Encuentros etnográficos con niños, niñas y adolescentes, editado por Alba Lucy Guerrero, Ángeles Clemente, Diana Milstein \& Maria Dantas-Whitney. magis, Revista Internacional de Investigación en Educación, 14, 1-6. doi: 10.11144/Javeriana.m14.rlbl

Barley, R. (2020). "Why have you not written my name?": Collaborative research with children. magis, Revista Internacional de Investigación en Educación, 13, 1-21. doi: 10.11144/Javeriana.m13.whwn

Dantas-Whitney, M. (2020). "Comimos quesadillas y después jugamos tag:" Children's bilingual/bicultural identity in a U. S. elementary school. magis, Revista Internacional de Investigación en Educación, 13, 1-21. doi: 10.11144/ Javeriana.m13.cqdj

Di Caudo-Villoslada, M. V. (2020). "No estamos de acuerdo con el título". Escribir un libro con estudiantes universitarios interculturales. magis, Revista Internacional de Investigación en Educación, 13, 1-27. doi: 10.11144/Javeriana. m13.neat 
Fernández, S. del C. (2021). "iEs porque no le ponen onda!": prácticas de comensalidad en la escuela. magis, Revista Internacional de Investigación en Educación, 14, 1-25. doi: 10.11144/Javeriana.m14.epnp

Guerrero, A. L., Clemente, A., Dantas-Whitney, M. \& Milstein, D. (2017). Bordes, límites y fronteras. Etnografía en colaboración con niños, niñas, adolescentes y jóvenes. Bogotá: Editorial Pontificia Universidad Javeriana.

Holmes, C. (2020). Childhood, play and school: A literature review in Australia. magis, Revista Internacional de Investigación en Educación, 13, 1-25. doi: 10.11144/Javeriana.m13.cpsl

Huerta-Cordova, V., Clemente-Olmos, A. \& Córdova-Hernández, L. (2021). El contexto vivo: reflexiones sobre una experiencia etnográfica colaborativa universitaria. magis, Revista Internacional de Investigación en Educación, 14, 1-20. doi: 10.11144/Javeriana.m14.cvre

Machado e Silva, R. C. (2021). "A mala não é dela": saber das crianças em contexto escolar de fronteira. magis, Revista Internacional de Investigación en Educación, 14, 1-33. doi: 10.11144/Javeriana.m14.mned

Milstein, D., Clemente, A. \& Guerrero, A. L. (2019). Collaboration in educational ethnography in education. En G. W. Noblit (Ed.), Oxford Research Encyclopedia, Education (oxfordre.com/education). Nueva York, NY: Oxford University Press.

Milstein, D. J., \& Guerrero, A. L. (2021). Lecturas de etnografías colaborativas con niñas, niños y jóvenes en contextos educativos latinoamericanos. magis, Revista Internacional de Investigación en Educación, 14, 1-33. doi: 10.11144/ Javeriana.m14.lecn

Rappaport, J. (2004). Más allá de la escritura: la epistemología de la etnografía en colaboración. Buenos Aires: Grupo de Apoyo Jurídico por el Acceso a la Tierra (Gajat). Recuperado de http://www.redalyc.org/pdf/1050/ 105015277007.pdf

Ruiz-Morales, M. M. (2021). "Nos convidamos solos en un cuento que no era nuestro": encuentros con niños investigadores. magis, Revista Internacional de Investigación en Educación, 14, 1-27. doi: 10.11144/Javeriana.m14.ncsc 